\title{
Correction to: Esophageal Clearance in Gastroesophageal Reflux
}

Maheen Hassan, Frederick W. Woodley, and Hayat Mousa

\section{Correction to: \\ Chapter 3 in: Y. Vandenplas (ed.), Gastroesophageal Reflux in Children, https://doi.org/10.1007/978-3-319-60678-1_3}

We received following corrections from author after publication of this book.

The name of an author was spelt incorrectly as Frederick W. Woodey in original version. His name has been corrected to Frederick W. Woodley throughout the book.

The updated original online version for this chapter can be found at https://doi.org/10.1007/978-3-319-60678-1_3 